\title{
Stars of the screen
}

\section{The latest releases for high-throughput analysis.}

\section{PrepExpress}

\section{Applied Biosystems}

www.appliedbiosystems.com

High-throughput compound characterization

Designed for the API 150EX PrepLC/MS system, the Windows-based PrepExpress application for Analyst software will automatically purify hundreds of compounds weekly for combinatorial and medicinal chemistry applications. A prescreening analytical experiment determines crude sample purity, and the software provides automatic submission of impure compounds for preparative purification, as well as automatic purity analysis of the collected fractions. The API 150EX PrepLC/MS system is configured in both analytical and preparative modes, with two Shimadzu LC8-A liquid chromatography pumps and a Gilson 215 autosampler/fraction collector.

\section{Tissue microarrays}

InnoGenex www.innogenex.com

Screening in situ gene expression

InnoGenex now produces tissue microarrays for high-throughput screening of in situ gene expression for target validation. Tissue microarrays are available from diseased and normal human tissues, mouse normal tissues and rat normal tissues. They are suitable for rapid screening of in situ differential expression of genes, identifying novel antibody markers, animal model studies, or for any other application that would use traditional tissue sections. Low-density arrays contain 20-50 elements per slide and high-density arrays have 100-200 elements per slide. First to be available in this new range are tissue arrays with normal and diseased lung, normal and diseased oesophagus, normal and diseased cervix, normal and diseased colon, normal human tissue arrays, and rat and mouse normal visceral organs for whole animal survey.

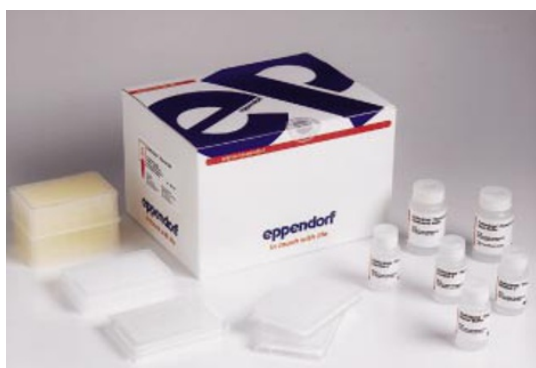

Eppendorf: Perfect for plasmid purification.

\section{Perfectprep Plasmid 384 Kit}

Eppendorf Plasmids for high throughput

The Perfectprep Plasmid 384 kit was developed for the high-throughput purification of plasmids using a liquid handling workstation. By using technology that ensures a high surface area, the kit overcomes the problems usually associated with the 384-well format and generates consistent high yields and optimal concentrations. The average yield with the Perfectprep Plasmid 384 kit is $1 \mu \mathrm{g}$ of highcopy plasmid from only $0.3 \mathrm{ml}$ of bacterial culture. Since the elution volume is only $10 \mu \mathrm{l}$, the average concentration is $100 \mathrm{ng}$ per $\mu \mathrm{l}$. The kit protocol features full 384-well format processing starting with bacteria cultivation (96-well cultivation is an option).

\section{MultiScreen Caco-2 Assay}

Millipore www.millipore.com Higher throughput ADME screening

Millipore's MultiScreen Caco-2 assay system performs in vitro Caco-2 drug transport assays. The system is validated and quality control released with Caco- 2 cell lines in 10day and 21-day cultures. Active drug screening results correlate with 24-well systems and meet FDA values for known compounds.
Optimized to grow and sustain high-integrity Caco-2 cell monolayers, the MultiScreen Caco-2 assay includes the necessary growth plates, feeding tray and transport analysis plate to complete the process from growth to analysis in one plate.

\section{ShortFast HPLC columns}

\section{Grace Vydac} www.grace.com High-throughput analyses in protein/peptide research

This new line of rapid analysis HPLC columns was developed for rapid separation of proteins and peptides. Applications include reaction kinetics, combinatorial library screening and proteomics. The makers say that ShortFast columns allow purification of more material, more quickly than ever before. Compared with analysis using conventional chromatography columns, ShortFast columns are claimed to reduce run times by as much as $85 \%$, and they also require less solvent usage, resulting in disposal cost savings.

\section{SNPCube}

Protedyne www.protedyne.com High-throughput SNP genotyping

The SNPCube automated high-throughput SNP genotyping system completely automates single nucleotide polymorphism (SNP) assays based on the TaqMan series of assays from Applied Biosystems. Included in the range is the SNPCube-2 system, incorporating assay preparation, multiplexed PCR thermal cycling, sequence detection, complete data tracking and data integration with Protedyne's XML LIMS interfacelayer. Speeds of up to 90,000 SNP reactions per day can be achieved.

These notes are compiled in the Nature office from information provided by the manufacturers. 